\title{
Wireless Sensor Network for Radiation Detection
}

\author{
Moez Altayeb ${ }^{1}$, Suliman Abdalla ${ }^{{ }^{*}}$, Marwa Mekki $^{2}$ \\ ${ }^{1}$ Instrumentation Centre, Sudan Atomic Energy Commission, P.O.Box 3001 Khartoum - Sudan \\ ${ }^{2}$ Faculty of Engineering and Technology, University of Gezira \\ e-mail:mohedahmed@hotmail.com,Suliman2003@hotmail.com*
}

\begin{abstract}
In this paper a wireless sensor network (WSN) is designed from a group of radiation detector stations with different types of sensors. These stations are located in different areas and each sensor transmits its data through GSM network to the main monitoring and control station. The design includes GPS module to determine the location of mobile and fixed station. The data is transmitted with GSM/GPRS modem. Instead of using traditional SMS data string or word messages a digital data frame is constructed and transmitted as SMS data. In the main monitoring station graphical user interface (GUI) software is designed to shows information and statues of the all stations in the network. It reports any radiation leaks, in addition to the data; the GUI contains a geographical map to display the location of the leakage station and can control the stations power consumption by sending a special command to it.
\end{abstract}

Keywords: radiation, GSM, GPS, GUI, GSM/GPRS, WSN

\section{Introduction}

The monitoring of radiation sources becomes a very important job now a day. The use of radiation sources is increased on several and many ways on our life. For instance it used for testing and quality purposes on industrial and manufacturing. In medical it used for treating, physical testing and experimenting. In addition, it also used in electric power reactors and many other ways. These radiation sources can cause some quantity of leaks during direct use or storage. These leakages can make a very dangerous physical injury for human and others. Electronic radiation sensors are a good solution for this problem but it is difficult to use and monitor separately.

To simplify this, a wireless sensor network is a power full solution. Radiation sensors are connected in nodes and passing its data to a main monitoring and control station through a wireless network. This technique makes the control of radiation leak more perfect and easy. Recent advances in micro-electro-mechanical systems (MEMS) technology, wireless communications, and digital electronics have enabled the development of low-cost, low-power, multifunctional sensor nodes that are small in size and communicate undeterred in short distances.

These tiny sensor nodes, which consist of sensing, data processing, and communicating components, leverage the idea of sensor networks based on collaborative effort of a large number of nodes. Sensor networks represent a significant improvement over traditional sensors, which are deployed in the following two ways [1]; the first sensors can be positioned far from the actual phenomenon, i.e., something known by sense perception. In this approach, large sensors that use some complex techniques to distinguish the targets from environmental noise are required. The second approach is several sensors that perform only sensing can be deployed. The positions of the sensors and communications topology are carefully engineered. They transmit time series of the sensed phenomenon to the central nodes where computations are performed and data are fused. The more modern networks are bidirectional, also enabling control of sensor activity. The development of wireless sensor networks was motivated by military applications such as battlefield surveillance; today such networks are used in many industrial and consumer applications, such as environmental monitoring, industrial process monitoring and control, machine health monitoring, and so on[2].

In general, within the sensor field, WSNs employ random-access channel sharing and transmission techniques that are now incorporated in the IEEE 802 family of standards; indeed, these techniques were originally developed in the late 1960s and 1970s expressly for wireless

Received September 29, 2016; Revised January 23, 2017; Accepted February 8, 2017 
environments and for large sets of dispersed nodes with limited channel-management intelligence. However, other channel management techniques are also available [3].

The WSN is built of nodes, from a few to several hundreds or even thousands, where each node is connected to one or sometimes several sensors. Each such sensor network node has typically several parts: a radio transceiver with an internal antenna or connection to an external antenna, a microcontroller, an electronic circuit for interfacing with the sensors and an energy source, usually a battery or an embedded form of energy harvesting. A sensor node might vary in size from that of a shoebox down to the size of a grain of dust, although functioning "motes" (sensor node and wireless node) of genuine microscopic dimensions have not yet to be created. The cost of sensor nodes is similarly variable, ranging from a few to hundreds of dollars, depending on the complexity of the individual sensor nodes. Size and cost constraints on sensor nodes result in corresponding constraints on resources such as energy, memory, computational speed and communications bandwidth[4].

\section{Methodology}

\subsection{Sensor Nodes}

Each sensor node has a sensor which can be GM tube, PIN diode, and photodiode. The function of the sensor is to detect radiations leakage on its position .The sensors are connected to their conditioning circuits and the ARDUINO UNO R3 microcontroller [5] is used to receive the signal from sensors and sends a warning message to the monitoring station through the GSM/GPRS modem [6]. To save the power on the sensor stations, the modem is set on power off state when there is no radiation detected. Figure 1 shows the block diagram of the WSN.

\subsection{Additional Nodes}

To monitor two or more radiation sources in the same station location, a mini sensor nodes that has the same components except the GPRS/GSM modem is used. These nodes monitor and control the stock which is used to store the radiation sources. Such sensor nodes include smoke detector, temperature sensor, water leaks, and water tank to be used for safety purposes. They are controlled by (PLC) system.

\subsection{Data Processing}

Data is sent to the main station as GSM message format. All nodes data is grouped in a frame with special format. The frame contains three fields the first field is station information (station number or name) with tow digit flowed by second field station location then third field three bits for sensors status. The frame can contain others fields as the design of the node require . The microcontroller software takes the location data from the GPS module [7] and put it in its field in SMS frame. The GPS system is useful in cases that the sensor station is not fixed on its position (automobile). Figure2 shows an aautomotive station with GPS module. The frame is sends to a computer where a software function processes it and extracts its data and displays it in a Graphical User Interface (GUI).In addition to the software process there is a database on the monitoring station. The database contains information about each node on the network such as (station hardware, communication type, and sensor detector type). 


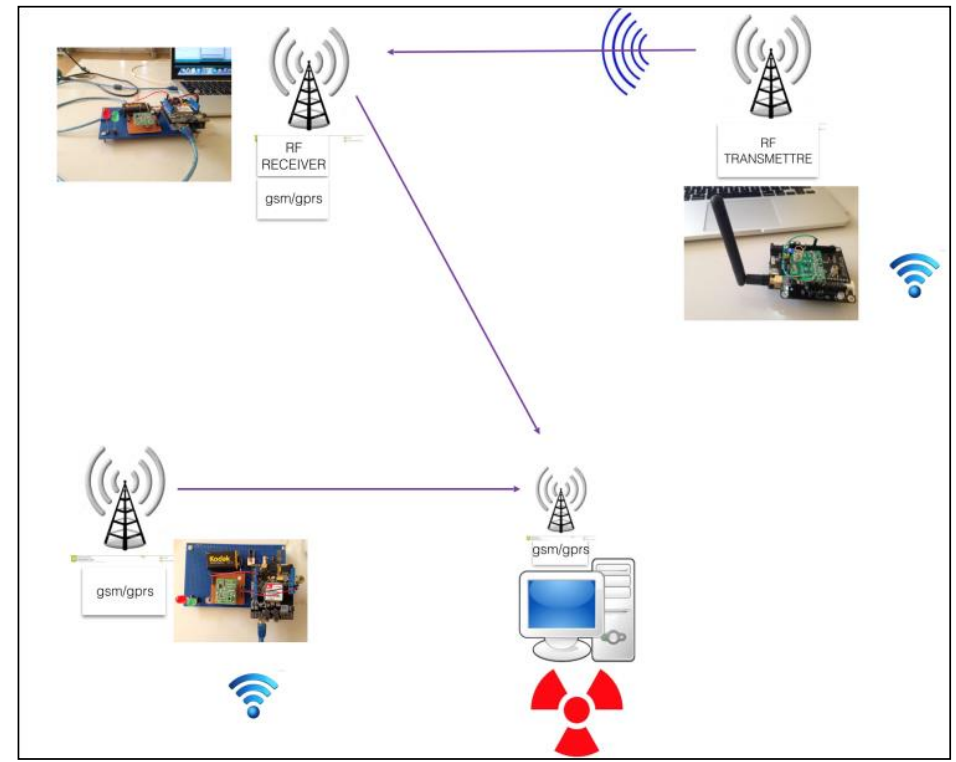

Figure 1. The block diagram of WSN

\subsection{Connection and Communication}

The connection between the sensors and their node is done by using microcontroller board and the communication between any node and the main station is done through the GSM/GPRS network. In the mini stations, the communication

between the sensors in the same station is done by RF transceiver [8], then the microcontroller in the sensor station send a frame of data to the sensor node. Figure3 illustrates the communication between sensor stations and GSM network.

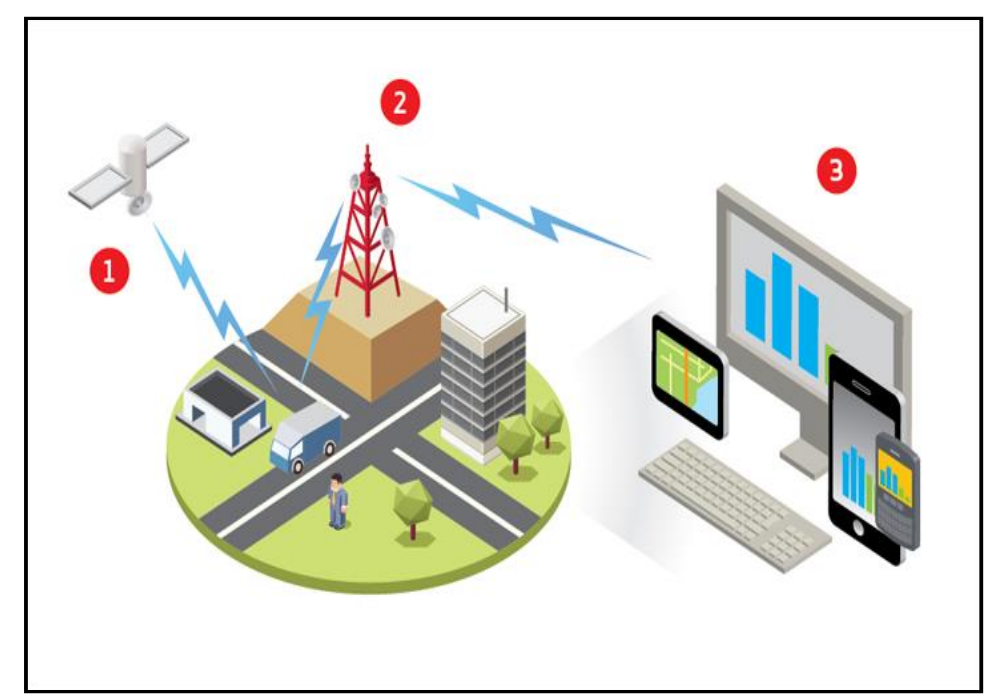

Figure 2. Automotive station with GPS module (1:GPS satellite,2:GSM,network 3:monotring station) 


\section{Design and Results}

\subsection{The Control Board}

To achieve signal processing and data transmitting on all stations, ARDUINO UNO R3 microcontroller board is used. The board is suitable because it has a built-in ADC used to receive data from sensors. More than one sensor can be connected the microcontroller. The microcontroller then sends a frame of data in the form of GSM SMS to the main station board.

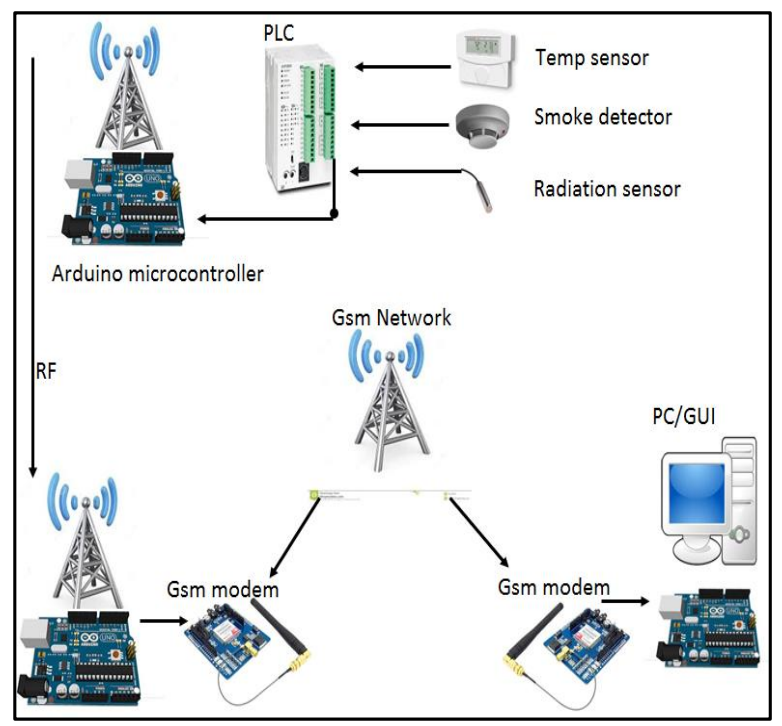

Figure 3. Sensor station (MICRO, GPRS/GSM, PHOTO DIOED)

\subsection{SMS Frame Construction}

The microcontroller sends a frame of data through RF system to the sensor station with GPRS/GSM modem . The connection between the two sensor nodes in the same station is done by RF transceiver. The sensor node with the GSM/GPRS modem is responded to send its data and all other data to the main monitoring station.

The most important data in the network is the radiation sensor data bit. When the microcontroller receive a radiation signal on its ADC channel port, it sets the radiation bit on the frame to 1 then it checks the smoke detector statues from its input port which connected to the PLC. If the input is 1 , that means the PLC report a fire condition, this makes the microcontroller to set the smoke bit of the frame to 1 . If the input from the PLC is 0 the microcontroller keeps the default value on the frame in 0 . For setting the location bits on the frame to the right value the microcontroller sends command to the GPS system and the module response by sending a frame through the UART. The microcontroller takes the location data from the GPS frame and put it on its position in SMS frame. The GPS system is useful when the sensor station is not on a fixed position.

The temperature sensor can be used in two ways: if it connected directly to the micro controller then its value in the SMS frame will be the real station temperature and if it connected to the PLC it will be adjusted to limited temperature value. Then its normal open switch closed when the station temperature reaches this value. In this case its bits in the SMS frame will set to 1. Finally the microcontroller put the station number on the first two bits and the frame is completed and ready for transmission. Figure 4 shows an Example of SMS station frame in normal condition.

The microcontroller sends a pulse on its output port to the modem to power it and this will take only one second. After the modem powered on the microcontroller sends the frame to it as SMS the modem delivers the SMS to the destination station through the GSM network.

IJEEI Vol. 5, No. 1, March 2017: $37-43$ 


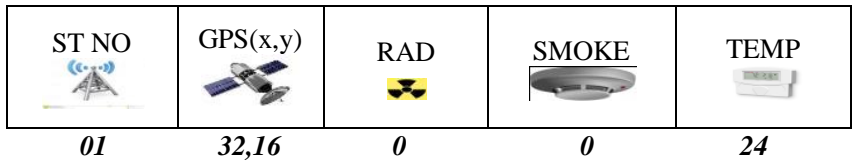

Figure 4: Example of SMS station frame in normal condition

\subsection{GUI Design}

When the frame is received in the monitoring station, software functions extract its data and process it and passed the processed data to the GUI window. The main monitoring and control station hardware consists of microcontroller and GSM modem. Figure 5 explains a monitoring station hardware and computer GUI . The GUI receives and sends data from and to the hardware with the universal a synchronous receiver transmitter (UART). The design of graphical user interface GUI is useful on displaying all information and data about all of the network nodes. It contains several software functions each of them has specific job. Start connect function goal is to open a port in the computer and make configuration for its property with special setting and connect it with the microcontroller port. Other function used to receive data from the modem and process it. An a functions used for displaying the processed data like location data in the map and radiation warning signal as red blinking square.

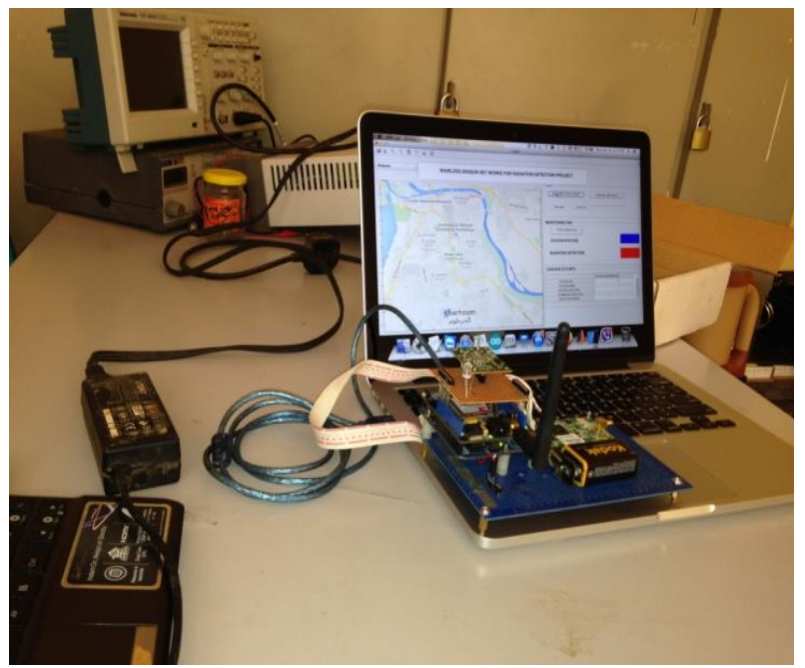

Figure 5. Monitoring station hardware and computer GUI

The GUI window also contains a control field with optional services like sending SMS warning message or a stored voice call to any cell phone number. The software function of this field sends special AT modem command [4] with the input phone number from the GUI input field to the GSM modem. Other optional service is the fire fighting system (water pumps) on the remote sensor station can be direct accessed by the GUI in case of automatic system failure figure 6 shows the window of the GUI with the output data which is get when test the remote sensor station. The red txet and smal circle in the map represents the location of the tested station, this location wil still blinking with animation until it cancelled by the user. The small table shwos all the data of the tested station. All the data and information in the GUI window can be genrated as areport file by the software 


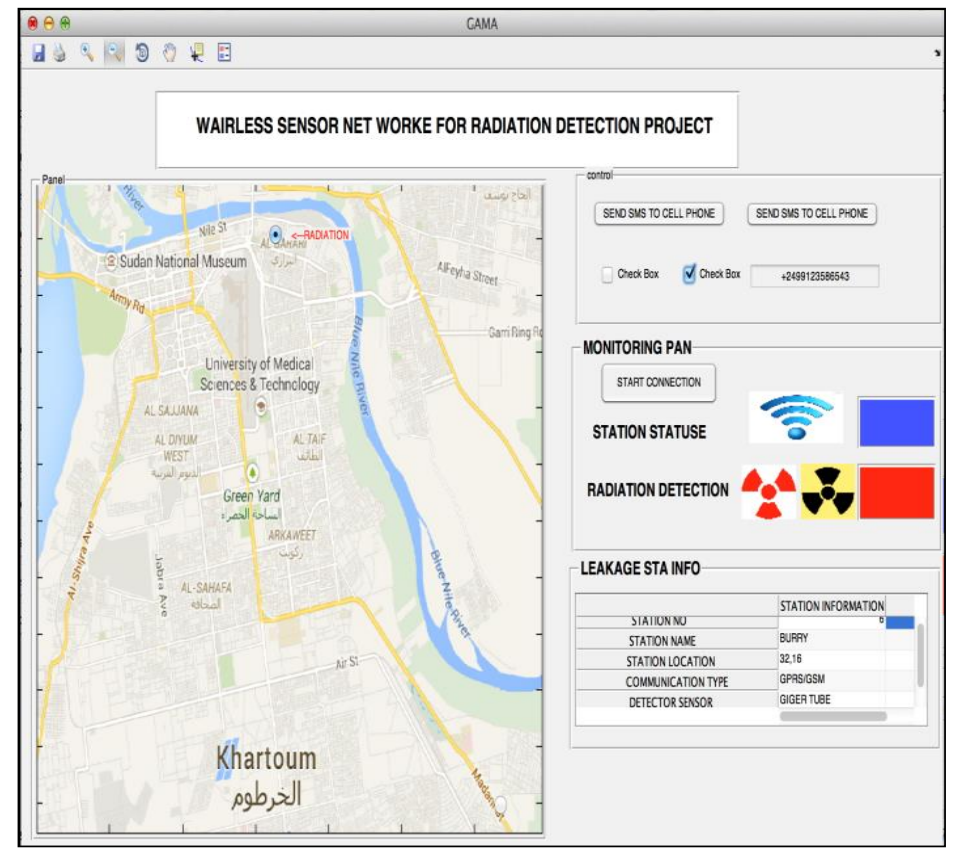

Figure 6. the GUI window in the monitor station

\subsection{Power Consumption Monitoring}

The microcontroller in the sensor station sends a signal to power on the modem then it sends the warning message. After the message received by the monitoring station in the destination the monitoring station send back a message to the sensor station that the leak is detected and to power off its modem to save its power figure 7 shows circuit's current in Amp before and during modem on/off condition . The signal under the current is the pulse introduced by the microcontroller to power on/off the modem. The duration of the pulse is one second, the vertical line represents the current in AMP and the horizontal line represents time in seconds.

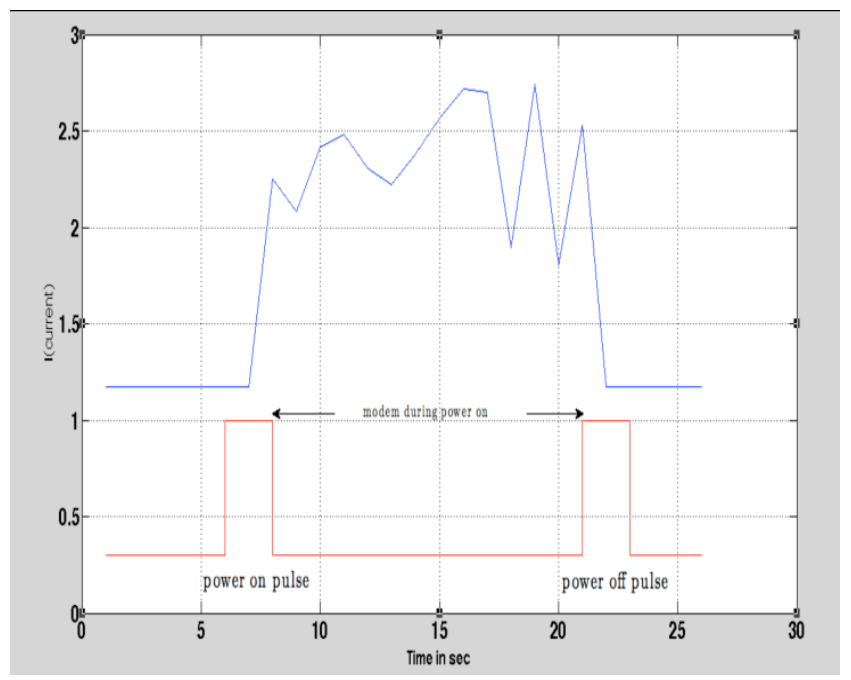

Figure 7. Circuit's current in Amp before and during modem on/off condition 


\section{Conclusions}

The task of this project is to demonstrate a self-powered wireless radiation detector with global communication capabilities. The proposed wireless detectors can operate independent of maintenance and human intervention for several years. Low cost and high spatial resolution can be achieved. To verify the concept of the proposed wireless detectors a field test will be performed and a sensor network will be deployed at sites of national interest.

\section{References}

[1] C Intanagonwiwat, R.Govindan, D Estrin. Directed diffusion: a scalable and robust communication paradigm for sensor networks. Proceedings of the ACM Mobi-Com'00. Boston. 2000: 56-67.

[2] K Martinez, P Padhy, A Riddoch, HL Ong, JK Hart. Glacial Environment Monitoring using Sensor Networks.

[3] Alan Mainwaring, Joseph Polastre, Robert Szewczyk, David Culler, John Anderson. Wireless Sensor Networks for Habitat Monitoring.

[4] KA Delin, SP Jackson, DW Johnson, SC Burleigh, RR Woodrow, M McAuley, JT Britton, JM Dohm, TPA Ferré, Felipe Ip, DF Rucker, and VR Baker. Sensor Web for Spatio-Temporal Monitoring of a Hydrological Environmental. 35th Lunar and Planetary Science Conference. 2004.

[5] http://arduino.cc/en/Main/ArduinoBoardUno.

[6] http://www.seeedstudio.com/depot/gprs-shield-p-779.html

[7] http://www.trimble.com/restime. RESOLUTION T SYSTEM DESIGNER REFERENCE MANUAL.

[8] FreakLabs. FREAKDUINO $900 \mathrm{MHz}$ Long Range Wireless Arduino-Compatible Prototyping Platform v2.1aDatasheet. 